\title{
STABILITY OF SURFACE MOTION ON A ROTATING ELLIPSOID
}

\author{
V. GUIBOUT ${ }^{1}$ and D. J. SCHEERES ${ }^{2}$ \\ ${ }^{1}$ Mechanical Department, Ecole Polytechnique, 91128 Palaiseau Cedex, France \\ ${ }^{2}$ Aerospace Engineering Department, University of Michigan, 1320 Beal Avenue, \\ Ann Arbor, MI 48109-2140, U.S.A.
}

(Received: 8 May 2002; revised: 19 December 2002; accepted: 12 March 2003)

\begin{abstract}
The dynamical environment on the surface of a rotating, massive ellipsoid is studied, with applications to surface motion on an asteroid. The analysis is performed using a combination of classical dynamics and geometrical analysis. Due to the small sizes of most asteroids, their shapes tend to differ from the classical spheroids found for the planets. The tri-axial ellipsoid model provides a non-trivial approximation of the gravitational potential of an asteroid and is amenable to analytical computation. Using this model, we study some properties of motion on the surface of an asteroid. We find all the equilibrium points on the surface of a rotating ellipsoid and we show that the stability of these points is intimately tied to the conditions for a Jacobi or MacLaurin ellipsoid of equilibria. Using geometrical analysis we can define global constraints on motion as a function of shape, rotation rate, and density, we find that some asteroids should have accumulation of material at their ends, while others should have accumulation of surface material at their poles. This study has implications for motion of a rover on an asteroid, and for the distribution of natural material on asteroids, and for a spacecraft hovering over an asteroid.
\end{abstract}

Key words: ellipsoid, asteroid, equilibrium point, stability

\section{Introduction}

The motivation for this paper is to better understand the motion of a particle traveling on the surface of an asteroid. A better understanding of this question has applications to the motion of natural material on the surface of an asteroid [9] and to the dynamics and control of an artificial object traveling on the surface of an asteroid [10]. Due to their small size and possible rapid rotation rates the combination of centripetal and gravitational accelerations change the dynamical environment on an asteroid surface to the point where simple models no longer apply, and a more sophisticated investigation must be used.

Due to the small size of most asteroids, their shapes tend to differ markedly from the classical spheroidal shapes found for planets. The general problem of surface motion on an asteroid must be analyzed with both a model for the asteroid surface and a model for the asteroid gravity field. While a spherical harmonic expansion 
model could be used for the shape of the asteroid [16], a spherical harmonic gravity field could not be used for such a computation due to their strong divergence at the surface of an asteroid [15]. Alternately, a polyhedron shape could be used for both the asteroid surface and its gravity field, and indeed, simple slope calculations have been performed for asteroids using such a model $[6,8,12,13,16]$, however the generality of these models make it difficult to extract the qualitative principles of motion on such a body. Thus, in this paper we adopt the tri-axial ellipsoid model to approximate an asteroid and its surface. There are three basic reasons that justify the use of this model. First because of its generality, as it can generate a wide range of shapes by adjusting only three parameters. Second because of its tractability, as its gravity field is known in closed form. Third because of its simple specification of the asteroid surface, which can be represented as a quadratic form. The ellipsoid model has also been used previously to study dynamics in the vicinity of asteroids $[2,11]$, and serves, in general, as the simplest non-trivial model for motion about such bodies. While the use of an ellipsoid model is clearly a strong approximation to a true asteroid shape, nonetheless we find that this provides a rich dynamical environment which can serve as the initial step towards understanding motion on the surface of more general asteroid shapes.

It should be stressed that the current study is not directly connected to the well-known problem of geodesic motion on the surface of an ellipsoid. In that problem the surface curvature acts as the force environment for the motion of the particle. In the problem considered here, the ellipsoid is not only rotating but is also gravitating, creating a fundamentally different environment than that considered in the study of geodesics, and preventing any direct comparisons between the two. One interesting aspect of our study is that we find a fundamental relationship between the stability of a particle resting on the surface of a rotating ellipsoid and the families of Jacobi and MacLaurin ellipsoids (see [1] for a complete review of these families).

The content of this paper is as follows. First we define the basic model and introduce normalizations to reduce the equations to their simplest form. Next we develop the conditions for equilibrium on the surface of a rotating ellipsoid. In general an ellipsoid will only have six unique equilibrium points, but we also find situations where every point on the surface of an ellipsoid may be in equilibrium. Following this we evaluate the stability of these equilibrium points using two different methods, first by performing a linear analysis about the equilibrium points and second by analyzing the zero-velocity curves associated with these points. Then we study the stability conditions and show that they are intimately related to the well-known families of Jacobi and MacLaurin ellipsoids. Finally, we apply our results to some known asteroids, showing that a variety of surface stability conditions probably exist in the solar system. 


\section{Description of the Tri-axial Ellipsoid Model}

\subsection{PHYSICAL CHARACTERISTICS}

To model an asteroid using a tri-axial ellipsoid we only need five parameters, the three major axes, the rotation rate and the density. In all the following, we assume that the tri-axial ellipsoid has semi-axes $\alpha, \beta$ and $\gamma$ with $\alpha \geqslant \beta \geqslant \gamma$, a constant density $\rho$, and a rotation rate $\omega$ about its maximum moment of inertia ( $\gamma$-axis). The gravitational parameter $\mu$ is then:

$$
\mu=\frac{4}{3} \pi G \rho \alpha \beta \gamma
$$

where $G$ is the gravitational constant and $4 \pi / 3 \rho \alpha \beta \gamma$ is the mass of the ellipsoid.

Now define a body-fixed coordinate system in the ellipsoid. The $x$-axis lies along the largest dimension $\alpha$, the $y$-axis lies along its intermediate dimension $\beta$ and the $z$-axis lies along its smallest dimension $\gamma$.

\subsection{GRAVITATIONAL POTENTIAL}

The gravitational potential on the surface of a constant density tri-axial ellipsoid is [7]

$$
V(x, y, z)=\frac{3 \mu}{4} \int_{0}^{\infty} \phi(x, y, z, u) \frac{\mathrm{d} u}{\Delta(u)}
$$

with

$$
\begin{aligned}
& \phi(x, y, z, u)=\frac{x^{2}}{\alpha^{2}+u}+\frac{y^{2}}{\beta^{2}+u}+\frac{z^{2}}{\gamma^{2}+u}-1, \\
& \Delta(u)=\sqrt{\left(\alpha^{2}+u\right)\left(\beta^{2}+u\right)\left(\gamma^{2}+u\right)}
\end{aligned}
$$

\subsection{SURFACE CONSTRAINT}

As mentioned previously, we are interested in motion on the surface. Thus the particle must satisfy the equation of the surface constraint:

$$
S(\mathbf{r})=0
$$

where

$$
S(\mathbf{r})=\frac{x^{2}}{\alpha^{2}}+\frac{y^{2}}{\beta^{2}}+\frac{z^{2}}{\gamma^{2}}-1 \quad \text { or } \quad S(\mathbf{r})=\phi(x, y, z, 0)
$$

Since motion is assumed to be tangent to the surface and the gradient of $S, \mathbf{S}_{r}$, is perpendicular to the surface, we have:

$$
\mathbf{S}_{r}=\left|S_{r}\right| \hat{n}, \quad \mathbf{S}_{r} \cdot \dot{\mathbf{r}}=0, \quad \mathbf{S}_{r} \cdot \ddot{\mathbf{r}}=0
$$

where $\hat{n}$ is the unit normal vector of the surface. 


\subsection{EQUATIONS OF MOTION}

The Lagrangian for unconstrained motion is stated as

$$
L=\frac{1}{2}(\dot{\mathbf{r}}+\boldsymbol{\omega} \wedge \mathbf{r}) \cdot(\dot{\mathbf{r}}+\boldsymbol{\omega} \wedge \mathbf{r})-V(\mathbf{r})
$$

To apply this to motion on the surface requires the Lagrangian to be augmented as

$$
L^{\prime}=L(\mathbf{r}, \dot{\mathbf{r}})+\lambda S(\mathbf{r})
$$

where $S(\mathbf{r})$ is the surface of the ellipsoid.

The equations of motion are then:

$$
\frac{\mathrm{d}}{\mathrm{d} t}\left(\frac{\partial L^{\prime}}{\partial \dot{\mathbf{r}}}\right)=\frac{\partial L^{\prime}}{\partial \mathbf{r}}, \quad 0=\frac{\partial L^{\prime}}{\partial \lambda}
$$

that is,

$$
\ddot{\mathbf{r}}+2 \omega \wedge \dot{\mathbf{r}}+\omega \wedge(\boldsymbol{\omega} \wedge \mathbf{r})=-\mathbf{V}_{r}+\lambda \mathbf{S}_{r}, \quad S(\mathbf{r})=0
$$

It is now useful to normalize all the variables:

$$
\hat{x}=\frac{x}{\alpha}, \quad \hat{y}=\frac{y}{\alpha}, \quad \hat{z}=\frac{z}{\alpha}, \quad \hat{t}=\omega t, \quad \delta=\frac{\mu}{\omega^{2} \alpha^{3}}
$$

The potential in the normalized coordinates is

$$
\hat{V}(\hat{x}, \hat{y}, \hat{z})=\frac{3 \delta}{4} \int_{0}^{\infty} \phi(\hat{x}, \hat{y}, \hat{z}, v) \frac{\mathrm{d} v}{\Delta(v)}
$$

where

$$
\begin{aligned}
& \phi(\hat{x}, \hat{y}, \hat{z}, v)=\frac{\hat{x}^{2}}{1+v}+\frac{\hat{y}^{2}}{\hat{\beta}^{2}+v}+\frac{\hat{z}^{2}}{\hat{\gamma}^{2}+v}-1, \\
& \Delta(v)=\sqrt{(1+v)\left(\hat{\beta}^{2}+v\right)\left(\hat{\gamma}^{2}+v\right),} \\
& \hat{\beta}=\frac{\beta}{\alpha} \quad \text { and } \quad \hat{\gamma}=\frac{\gamma}{\alpha}
\end{aligned}
$$

Expanding the integral yields:

$$
\hat{V}(\hat{x}, \hat{y}, \hat{z})=\frac{3}{4} \delta\left(I_{\hat{\alpha}} \hat{x}^{2}+I_{\hat{\beta}} \hat{y}^{2}+I_{\hat{\gamma}} \hat{z}^{2}-\hat{I}\right)
$$

where

$$
\begin{array}{ll}
I_{\hat{\alpha}}=\int_{0}^{\infty} \frac{\mathrm{d} u}{(1+u) \Delta(u)}, & I_{\hat{\beta}}=\int_{0}^{\infty} \frac{\mathrm{d} u}{\left(\hat{\beta}^{2}+u\right) \Delta(u)}, \\
I_{\hat{\gamma}}=\int_{0}^{\infty} \frac{\mathrm{d} u}{\left(\hat{\gamma}^{2}+u\right) \Delta(u)}, & \hat{I}=\int_{0}^{\infty} \frac{\mathrm{d} u}{\Delta(u)}
\end{array}
$$


Moreover, as $1 \geqslant \hat{\beta} \geqslant \hat{\gamma}$, it immediately follows that:

$$
I_{\hat{\alpha}} \leqslant I_{\hat{\beta}} \leqslant I_{\hat{\gamma}}, \quad I_{\hat{\alpha}} \geqslant \hat{\beta}^{2} I_{\hat{\beta}} \geqslant \hat{\gamma}^{2} I_{\hat{\gamma}}
$$

To state the final form of the equation, we drop the hat notation and assume all quantities to be normalized:

$$
\begin{aligned}
& \ddot{\mathbf{r}}+2 \boldsymbol{\omega} \wedge \dot{\mathbf{r}}+\boldsymbol{\omega} \wedge(\boldsymbol{\omega} \wedge \mathbf{r})=-\mathbf{V}_{r}+\lambda \mathbf{S}_{r}, \\
& S(\mathbf{r})=x^{2}+\frac{y^{2}}{\beta^{2}}+\frac{z^{2}}{\gamma^{2}}-1=0
\end{aligned}
$$

where $\omega$ has unit magnitude and points along the $\gamma$ axis.

Thus we have reduced the number of parameters from 5 to 3 ( $\beta, \gamma$ and $\delta$ ).

\subsection{EVALUATION OF THE LAGRANGE MULTIPLIER $\lambda$}

To simplify the full motion (Equation (12)) we must solve for the Lagrange multiplier $\lambda$ in terms of the positions and velocities of the particle. The dot product of $\mathbf{S}_{r}$ with Equation (12) yields:

$$
\mathbf{S}_{r} \cdot(\ddot{\mathbf{r}}+2 \boldsymbol{\omega} \wedge \dot{\mathbf{r}}+\boldsymbol{\omega} \wedge(\boldsymbol{\omega} \wedge \mathbf{r}))=\mathbf{S}_{r} \cdot\left(-\mathbf{V}_{r}+\lambda \mathbf{S}_{r}\right)
$$

Using Equations (4), we solve for $\lambda$ :

$$
\lambda\left|\mathbf{S}_{r}\right|^{2}=\mathbf{S}_{r} \cdot\left\{2 \boldsymbol{\omega} \wedge \dot{\mathbf{r}}+\boldsymbol{\omega} \wedge(\boldsymbol{\omega} \wedge \mathbf{r})+\mathbf{V}_{r}\right\}
$$

Using Equations (12) and (13) we are now able to study the existence of the equilibrium points.

\section{Existence of Equilibrium Points}

Substituting Equation (13) into Equation (12) and simplifying yields:

$$
\begin{aligned}
& \ddot{\mathbf{r}}+\mathcal{I}_{n n}[2 \boldsymbol{\omega} \wedge \dot{\mathbf{r}}]=\mathcal{I}_{n n}\left[-\boldsymbol{\omega} \wedge(\boldsymbol{\omega} \wedge \mathbf{r})-\mathbf{V}_{r}\right] \\
& S(\mathbf{r})=0
\end{aligned}
$$

where $\mathcal{I}_{n n}=\mathcal{I}-\left(\mathbf{S}_{r} /\left|\mathbf{S}_{r}\right|\right)\left(\mathbf{S}_{r} /\left|\mathbf{S}_{r}\right|\right)^{T}$ projects a general vector into the space tangent to the ellipsoid surface, and $\mathcal{I}$ is the identity matrix. 
We are interested in the equilibrium points, so we set $\dot{\mathbf{r}}=\ddot{\mathbf{r}}=0$. Simplifying and expanding yields:

$$
\begin{gathered}
x\left[1-\frac{4}{\left|\mathbf{S}_{r}\right|^{2}}\left(x^{2}+\frac{y^{2}}{\beta^{2}}\right)-\frac{3 \delta}{2} I_{\alpha}\right. \\
\left.+\frac{6 \delta}{\left|\mathbf{S}_{r}\right|^{2}}\left(x^{2} I_{\alpha}+\frac{y^{2}}{\beta^{2}} I_{\beta}+\frac{z^{2}}{\gamma^{2}} I_{\gamma}\right)\right]=0 \\
\frac{y}{\beta^{2}}\left[\beta^{2}-\frac{4}{\left|\mathbf{S}_{r}\right|^{2}}\left(x^{2}+\frac{y^{2}}{\beta^{2}}\right)-\frac{3 \delta}{2} \beta^{2} I_{\beta}\right. \\
\left.\quad+\frac{6 \delta}{\left|\mathbf{S}_{r}\right|^{2}}\left(x^{2} I_{\alpha}+\frac{y^{2}}{\beta^{2}} I_{\beta}+\frac{z^{2}}{\gamma^{2}} I_{\gamma}\right)\right]=0 \\
\frac{z}{\gamma^{2}}\left[\gamma^{2}-\frac{4}{\left|\mathbf{S}_{r}\right|^{2}}\left(x^{2}+\frac{y^{2}}{\beta^{2}}\right)-\frac{3 \delta}{2} \gamma^{2} I_{\gamma}\right. \\
\left.\quad+\frac{6 \delta}{\left|\mathbf{S}_{r}\right|^{2}}\left(x^{2} I_{\alpha}+\frac{y^{2}}{\beta^{2}} I_{\beta}+\frac{z^{2}}{\gamma^{2}} I_{\gamma}\right)\right]=0 \\
x^{2}+\frac{y^{2}}{\beta^{2}}+\frac{z^{2}}{\gamma^{2}}-1=0
\end{gathered}
$$

We immediately note that points $P_{1}=( \pm 1,0,0), P_{2}=(0, \pm \beta, 0)$ and $P_{3}=$ $(0,0, \pm \gamma)$ are equilibrium points, since they verify Equations (16)-(19).

Let us try to find other equilibrium points. For that let us first find some useful relationships. The difference between Equation (16) divided by $x$ and Equation (17) divided by $y / \beta^{2}$, the difference between Equation (16) divided by $x$ and Equation (18) divided by $z / \gamma^{2}$ and the difference between Equation (17) divided by $y / \beta^{2}$ and Equation (18) divided by $z / \gamma^{2}$ lead to:

$$
\begin{aligned}
& 1-\beta^{2}=\frac{3}{2} \delta\left(I_{\alpha}-\beta^{2} I_{\beta}\right) \\
& 1=\frac{3}{2} \delta\left(I_{\alpha}-\gamma^{2} I_{\gamma}\right) \\
& \beta^{2}=\frac{3}{2} \delta\left(\beta^{2} I_{\beta}-\gamma^{2} I_{\gamma}\right)
\end{aligned}
$$

Note that these equations are not necessarily true in general, they are verified only for equilibrium points, and thus will allow us to search for additional equilibrium points.

1. If $x \neq 0$ then

- if $y \neq 0$, Equation (17) can be replaced by Equation (20) which is a linear combination of Equations (17) and (16) (note that if $\beta=1$ this new equation becomes void).

- If $z \neq 0$, Equation (18) can be replaced by Equation (21). To find equilibrium points, we now need to solve Equations (16), (20), (21) and (19). 
Nevertheless, Equations (20) and (21) are only conditions on the physical characteristic of the ellipsoid, they define the Jacobi ellipsoid. ${ }^{1}$ If Equations (20) and (21) are satisfied (i.e. if we have a Jacobi ellipsoid), we substitute $z$ in Equation (16) using (19), and simplify using Equations (20) and (21) to find that all points on the ellipsoid surface (except the equator because we set $z \neq 0$ ) are solutions of our system, that is, are in equilibrium. If $\beta=1$, we have seen that Equation (20) is void. In that case, only Equation (21) describes the ellipsoid. Its solution is known as the MacLaurin spheroid. If it is satisfied, then again all points on the surface are equilibria. Finally, if $\gamma=1$, there is no solution to Equation (21) and there is no equilibrium point.

- If $z=0$, Equation (18) is always true. We only need to solve Equations (16), (20) and (19). If we substitute $y$ in Equation (16) using (19), and simplify using Equation (20), we find that all points on the equator are solutions, that is are in equilibrium. The geometry defined by Equation (20) includes, but is not limited to, the Jacobi ellipsoid and the MacLaurin spheroid.

- if $y=0$ Equation (17) is always true.

- $z=0$ leads to $P_{1}$

- If $z \neq 0$ we replace Equation (18) by Equation (21). We now have to solve Equations (16), (21) and (19). If we substitute $z$ in Equation (16) using (19), and simplify using Equation (21), we find that all points on the ellipse which lies on the ellipsoid in the plane $y=0$ are solutions, that is are in equilibrium. The geometry defined by (21) includes, but is not limited to, the Jacobi ellipsoid and the MacLaurin spheroid. Note that if $\gamma=1$ there is no solution to (21) and there is no equilibrium point.

2. If $x=0$, Equation (16) is always true

$-y=0$ leads to $P_{3}$,

$-y \neq 0$ and $z=0$ lead to $P_{2}$,

$-y \neq 0$ and $z \neq 0$ allows us to replace Equation (18) by Equation (22). If we substitute $z$ in Equation (17) using (19), and simplify using Equation (22), we find that all points on the ellipse which lies on the ellipsoid in the plane $x=0$ are solutions, that is are in equilibrium. The geometry defined by (22) includes, but is not limited to, the Jacobi ellipsoid and the MacLaurin spheroid.

Through this analysis, we have shown that in the most general case the only equilibrium points for $\beta<1$ are $P_{1}, P_{2}$ and $P_{3}$. Nevertheless, there exist some geometries for which the ellipsoid has infinitely many equilibrium points. The MacLaurin spheroids and the Jacobi ellipsoids are some well-known figures for

${ }^{1}$ Chandrasekhar [1] gives a complete description of this equilibrium figure and discusses some of their properties. The link between the Jacobi ellipsoids and our study is made in Appendix A. 
which all points on the surface are in equilibrium. For each $\beta$, there exists a unique $(\gamma, \delta)$ defining such a figure that is a solution to Equations (20)-(22). We also put into relief the existence of infinitely many geometries for which all points on the ellipses which lie in the plane $z=0$ or $y=0$ or $x=0$ are in equilibrium. These geometries are defined by satisfying only one equation among Equations (20)-(22).

We can already underline a very interesting property of the equilibrium points, for some particular values of $(\beta, \gamma, \delta)$ there exist an infinite number of equilibrium points, whereas in the other cases there are only six. Thus, for these special values, we expect a discontinuity in the properties of the six equilibrium points.

\section{Stability of the Equilibrium Points}

In this section we use Equations (12) and (13) to study the equilibrium points $P_{1}$, $P_{2}$ and $P_{3}$ found in the previous section. The first step will be to linearize the equations of motion about the equilibrium points and find the conditions for which they are a stable oscillator, that is for which we have spectral stability. Re-write Equations (12) and (13) in the coordinate system:

$$
\begin{aligned}
& \ddot{x}-2 \dot{y}=x\left(1-\frac{3 \delta}{2} I_{\alpha}+2 \lambda\right), \quad \ddot{y}+2 \dot{x}=y\left(1-\frac{3 \delta}{2} I_{\beta}+\frac{2 \lambda}{\beta^{2}}\right), \\
& \ddot{z}=z\left(-\frac{3 \delta}{2} I_{\gamma}+\frac{2 \lambda}{\gamma^{2}}\right) \\
& S(\mathbf{r})=0 \\
& \lambda=\frac{1}{2}\left(-2 x \dot{y}-x^{2}+\frac{3 \delta}{2}\left(x^{2} I_{\alpha}+\frac{y^{2}}{\beta^{2}} I_{\beta}+\frac{z^{2}}{\gamma^{2}} I_{\gamma}\right)+\frac{2 y \dot{x}}{\beta^{2}}-\frac{y^{2}}{\beta^{2}}\right)
\end{aligned}
$$

We will consider the stability of the points $P_{i}$ for the cases $\beta=1$ and $\beta \neq 1$ separately.

\subsection{STABILITY WHEN $\beta \neq 1$}

\subsubsection{Stability of $P_{1}$}

In the vicinity of $P_{1}$, we have: $x=1+\Delta x, y=\Delta y, z=\Delta z$ with $\Delta x, \Delta y$, $\Delta z \ll 1$.

First of all, we need to expand $\lambda$ around $P_{1}$ keeping only the terms of order 0 and 1 :

$$
\lambda=\frac{1}{2}\left(-1+\frac{3}{2} \delta I_{\alpha}-2 x \dot{y}\right)
$$


Now replace $\lambda$ in Equation (23) and only keep the terms of lower order:

$$
\begin{aligned}
& \ddot{x}=0 \\
& \ddot{y}+2 \dot{x}=y\left(1-\frac{3 \delta}{2} I_{\beta}+\frac{1}{\beta^{2}}\left(-1+\frac{3 \delta}{2} I_{\alpha}\right)\right) \\
& \ddot{z}=z\left(-\frac{3 \delta}{2} I_{\gamma}+\frac{1}{\gamma^{2}}\left(-1+\frac{3 \delta}{2} I_{\alpha}\right)\right)
\end{aligned}
$$

From Equation (24) we get

$$
2 \Delta x+\frac{\Delta y^{2}}{\beta^{2}}+\frac{\Delta z^{2}}{\gamma^{2}}=0
$$

Therefore, $\Delta x$ is of order $\Delta y^{2}$ and $\Delta z^{2}$ at least. We can then simplify Equation (28), since $\dot{x}$ is small with respect to $\ddot{y}$ :

$$
\ddot{y}=y\left[1-\frac{3 \delta}{2} I_{\beta}+\frac{1}{\beta^{2}}\left(-1+\frac{3 \delta}{2} I_{\alpha}\right)\right]
$$

$P_{1}$ is spectrally stable if this equation defines an oscillator. We must have:

$$
1-\frac{3 \delta}{2} I_{\beta}+\frac{1}{\beta^{2}}\left(-1+\frac{3 \delta}{2} I_{\alpha}\right)<0
$$

Let us call this Condition 1. We will study it later.

Equation (29) is also an oscillator if

$$
-\frac{3 \delta}{2} I_{\gamma}+\frac{1}{\gamma^{2}}\left(-1+\frac{3 \delta}{2} I_{\alpha}\right)<0
$$

We call this Condition 2.

Thus, $P_{1}$ is spectrally stable if and only if Conditions 1 and 2 are realized.

Finally, using the theory of the Lagrangian multiplier, we know that the particle stays on the surface of the ellipsoid if and only if the constraint $S(\mathbf{r})=0$ is active, that is, if $\lambda>0$. This inequality is a condition of existence for the equilibrium point $P_{1}$, it assures us that the centrifugal force is not too strong as compared to the gravitational force. ${ }^{2}$ We can re-write (26) to the zeroth order as $\delta>2 / 3 I_{\alpha}$.

\subsubsection{Stability of $P_{2}$}

At $P_{2}$, we have: $x=\Delta x, y=\beta+\Delta y, z=\Delta z$ with $\Delta x, \Delta y, \Delta z \ll 1$. Here the expansion of $\lambda$ in that case is

$$
\lambda=\frac{\beta^{2}}{2}\left(-1+\frac{3 \delta}{2} I_{\beta}+\frac{2 y \dot{x}}{\beta^{2}}\right)
$$

\footnotetext{
${ }^{2}$ If the centrifugal force is too strong, the particle cannot stay on the surface of the ellipsoid.
} 
Replacing $\lambda$ and keeping the terms of lowest order yields:

$$
\begin{aligned}
& \ddot{x}-2 \dot{y}=x\left[1-\frac{3 \delta}{2} I_{\alpha}+\beta^{2}\left(-1+\frac{3 \delta}{2} I_{\beta}\right)\right] \\
& \ddot{y}=0 \\
& \ddot{z}=z\left[-\frac{3 \delta}{2} I_{\gamma}+\frac{\beta^{2}}{\gamma^{2}}\left(-1+\frac{3 \delta}{2} I_{\beta}\right)\right]
\end{aligned}
$$

From Equation (24) we get

$$
\Delta x^{2}+2 \frac{\Delta y}{\beta}+\frac{\Delta z^{2}}{\gamma^{2}}=0
$$

Therefore, $\Delta y$ is of order $\Delta x^{2}$ and $\Delta z^{2}$ at least. We can then simplify the Equation (32):

$$
\ddot{x}=x\left(1-\frac{3 \delta}{2} I_{\alpha}+\beta^{2}\left(-1+\frac{3 \delta}{2} I_{\beta}\right)\right)
$$

This equation defines an oscillator if

$$
1-\frac{3 \delta}{2} I_{\alpha}+\beta^{2}\left(-1+\frac{3 \delta}{2} I_{\beta}\right)<0
$$

We call this Condition 3.

Equation (34) is also an oscillator if Condition 4 holds:

$$
-\frac{3 \delta}{2} I_{\gamma}+\frac{\beta^{2}}{\gamma^{2}}\left(-1+\frac{3 \delta}{2} I_{\beta}\right)<0
$$

To conclude, $P_{2}$ is spectrally stable if and only if Conditions 3 and 4 are realized.

Again, we know that the particle stays on the surface of the ellipsoid if and only if the constraint $S(\mathbf{r})=0$ is active, that is, if $\lambda>0$. This inequality is a condition of existence of the equilibrium point $P_{2}$. We can re-write it at the zeroth order as: $\delta>2 / 3 I_{\beta}$.

\subsubsection{Stability of $P_{3}$}

At $P_{3}$, we have: $x=\Delta x, y=\Delta y, z=\gamma+\Delta z$ with $\Delta x, \Delta y, \Delta z \ll 1$. In this case, the expansion of $\lambda$ is

$$
\lambda=\frac{\gamma^{2}}{2} \frac{3 \delta}{2} I_{\gamma}
$$

Equation (23) becomes:

$$
\begin{aligned}
& \ddot{x}-2 \dot{y}=x\left(1-\frac{3 \delta}{2} I_{\alpha}+\gamma^{2} \frac{3 \delta}{2} I_{\gamma}\right) \\
& \ddot{y}+2 \dot{x}=y\left(1-\frac{3 \delta}{2} I_{\beta}+\frac{\gamma^{2}}{\beta^{2}} \frac{3 \delta}{2} I_{\gamma}\right) \\
& \ddot{z}=0
\end{aligned}
$$


From Equation (24) we get

$$
\Delta x^{2}+\frac{\Delta y^{2}}{\beta^{2}}+2 \frac{\Delta z}{\gamma}=0
$$

Therefore, $\Delta z$ is of order $\Delta x^{2}$ and $\Delta y^{2}$ at least. $P_{3}$ is spectrally stable if and only if for $x=K \exp \lambda t$ and $y=L \exp \lambda t, \Re(\lambda)=0$. Replacing $x$ and $y$ in Equations (37) and (38) yields:

$$
\begin{aligned}
& K\left(\lambda^{2}-\left(1-\frac{3}{2} \delta I_{\alpha}+\gamma^{2} \frac{3}{2} \delta I_{\gamma}\right)\right)-2 L \lambda=0 \\
& 2 K \lambda+L\left(\lambda^{2}-\left(1-\frac{3}{2} \delta I_{\beta}+\gamma^{2} \frac{3}{2} \delta I_{\gamma}\right)\right)=0
\end{aligned}
$$

The condition for this system to have a solution is

$$
\lambda^{4}-\lambda^{2}(A+B-4)+A B=0
$$

where

$$
A=1-\frac{3 \delta}{2} I_{\alpha}+\gamma^{2} \frac{3 \delta}{2} I_{\gamma}, \quad B=1-\frac{3 \delta}{2} I_{\beta}+\frac{\gamma^{2}}{\beta^{2}} \frac{3 \delta}{2} I_{\gamma}
$$

Moreover, $\Re(\lambda)=0$ implies that

$$
A+B-4<0, \quad A B>0
$$

Thus we conclude that $A$ and $B$ must be negative (if $A<0$ and $B<0, A+B-4$ is always negative). We finally find Conditions 5 and 6 :

$$
\begin{aligned}
& \text { Condition } 5 \Leftrightarrow \frac{3}{2} \delta\left(I_{\alpha}-\gamma^{2} I_{\gamma}\right)>1, \\
& \text { Condition } 6 \Leftrightarrow \frac{3}{2} \delta\left(\beta^{2} I_{\beta}-\gamma^{2} I_{\gamma}\right)>\beta^{2}
\end{aligned}
$$

$P_{3}$ is spectrally stable if and only if Condition 5 and Condition 6 are realized.

Finally, we know that the particle stays on the surface of the ellipsoid if and only if the constraint $S(\mathbf{r})=0$ is active, that is, if $\lambda>0$. This is always true so the equilibrium point $P_{3}$ always exists.

\subsection{IF $\beta=1$}

The case $\beta=1$ corresponds to the ellipsoid axisymmetric with respect to the axis $z$, that is an oblate or a prolate spheroid. A rotation of the coordinate system around the $z$-axis does not influence either of the Equations (23) and (25). So we can study the stability of any point on the equator. Let us choose the point $(1,0,0)$. A limited 
development of $\lambda$ gives:

$$
\lambda=\frac{1}{2}\left(-1+\frac{3}{2} \delta I_{\alpha}-2 x \dot{y}\right)
$$

We replace $\lambda$ in the equations of motion (23) and keep the terms of the lowest order:

$$
\begin{aligned}
& \ddot{x}=0 \\
& \ddot{y}+2 \dot{x}=-2 y \dot{y} \\
& \ddot{z}=z\left(-\frac{3 \delta}{2} I_{\gamma}+\frac{1}{\gamma^{2}}\left(-1+\frac{3 \delta}{2} I_{\alpha}\right)\right)
\end{aligned}
$$

Equation (42) shows that $\Delta x$ is of second order. In Equation (43), $y \dot{y}$ is a term of the second order, so $\ddot{y}$ is also of second order. Therefore, to the first order,

$$
\ddot{y}=\ddot{x}=0 \quad \text { and } \quad \dot{y} \neq 0
$$

The particle will have a constant speed along the $x$-axis and $y$-axis. Thus, in the case $\beta=1$, the points on the equator are always unstable.

Concerning $P_{3}$, the resolution is the same as before. However, Condition 5 and Condition 6 are identical for $\beta=1$.

\subsection{THE OTHER EQUILIBRIUM POINTS}

If the ellipsoid satisfies either the Jacobi ellipsoid conditions or the MacLaurin spheroid conditions, then the entire ellipsoid surface is an equilibrium position. Therefore, all points are unstable in that a particle perturbed with a small velocity will maintain that velocity in the ensuing motion.

If the ellipsoid satisfies only one condition among the three defined by Equations (20)-(22), then there are infinitely many equilibrium points which lie on an ellipse along one of the symmetries $x=0, y=0$ or $z=0$. Therefore, all points are unstable in that a particle perturbed with a small velocity tangent to the ellipse will maintain that velocity in the ensuing motion.

In summary, we have found the conditions of spectral stability for all the equilibrium points. For $P_{1}, P_{2}$ and $P_{3}$ there are two conditions (sometimes the same) for stability and one condition for existence. Considering the linearized system, the condition for existence can be viewed as a condition for stability along the axis on which lies the equilibrium points whereas the two conditions of stability describe the stability along the two other axes. For instance, if Condition 2 is true, $P_{1}$ is stable along the $z$-axis but if Condition 1 is not true, $P_{1}$ is not stable along the $y$-axis. The condition of existence characterizes the stability along the $x$-axis. 
We will now expand these results to Hill stability (see [16]) with a more general method using quadratic forms.

\section{Studies of Stability Using Geometrical Reasoning}

\subsection{JACOBI INTEGRAL}

Taking the dot product of the equation of motion (12) by $\dot{r}$, and integrating yields:

$$
J=\frac{1}{2} \frac{\mathrm{d} \mathbf{r}}{\mathrm{d} t} \cdot \frac{\mathrm{d} \mathbf{r}}{\mathrm{d} t}-\frac{1}{2}(\boldsymbol{\omega} \wedge \mathbf{r}) \cdot(\boldsymbol{\omega} \wedge \mathbf{r})+V(\mathbf{r})
$$

since $\mathbf{S}_{r} \cdot \dot{\mathbf{r}}=0$. $J$ is a constant of the system called the Jacobi constant.

It can be expanded, replacing $V(\mathbf{r})$ by $(3 \delta / 4)\left(I_{\alpha} x^{2}+I_{\beta} y^{2}+I_{\gamma} z^{2}-I\right)$ :

$$
J=\frac{1}{2} \frac{\mathrm{d} \mathbf{r}}{\mathrm{d} t} \cdot \frac{\mathrm{d} \mathbf{r}}{\mathrm{d} t}-\frac{1}{2}\left(x^{2}+y^{2}\right)+\frac{3 \delta}{4}\left(I_{\alpha} x^{2}+I_{\beta} y^{2}+I_{\gamma} z^{2}-I\right)
$$

To study the stability of motion, we are interested in the zero-velocity curves on the surface of the ellipsoid.

If we suppose the speed relative to the surface is zero, the Jacobi equation becomes:

$$
J^{\prime}=x^{2}\left(-\frac{1}{2}+\frac{3}{4} \delta I_{\alpha}\right)+y^{2}\left(-\frac{1}{2}+\frac{3}{4} \delta I_{\beta}\right)+z^{2} \frac{3}{4} \delta I_{\gamma}
$$

where $J^{\prime}=J+(3 \delta / 4) I$.

It can be re written as

$$
J^{\prime}=J_{1}^{\prime} x^{2}+J_{2}^{\prime} y^{2}+J_{3}^{\prime} z^{2}
$$

where

$$
J_{1}^{\prime}=-\frac{1}{2}+\frac{3}{4} \delta I_{\alpha}, \quad J_{2}^{\prime}=-\frac{1}{2}+\frac{3}{4} \delta I_{\beta}, \quad J_{3}^{\prime}=\frac{3}{4} \delta I_{\gamma}
$$

Thus $J^{\prime}$ is a quadratic form, and motion is only allowed in the space

$$
J^{\prime} \geqslant J_{1}^{\prime} x^{2}+J_{2}^{\prime} y^{2}+J_{3}^{\prime} z^{2}
$$

Moreover, as the motion must lie on the surface of the ellipsoid, we have the additional constraint:

$$
S(x, y, z)=x^{2}+\frac{y^{2}}{\beta^{2}}+\frac{z^{2}}{\gamma^{2}}-1
$$

with $1 \geqslant \beta \geqslant \gamma>0$

The zero-velocity curves are found by solving the Jacobi equation (Equation (48)) under the constraint $S(\mathbf{r})=0$ (Equation (50)), that is, by finding the intersection of these two quadratic forms. 


\subsection{QUADRATIC FORMS}

Suppose we are at a given equilibrium point $P_{i}, i \in\{1,2,3\}$, with no speed, $\mathbf{v}=0$, then

$$
J^{\prime}=r_{i} J_{i}^{\prime}
$$

where $r_{1}=1, r_{2}=\beta^{2}$ or $r_{3}=\gamma^{2}$ depending on the equilibrium point we are interested in.

Now assume a speed $\Delta \mathbf{v}$ is given to the particle, and then let us find the zerovelocity curves. We have $J^{\prime}=r_{i} J_{i}^{\prime}+\Delta J^{\prime}$, where $\Delta J^{\prime}=(1 / 2) \Delta v^{2}$

Equation (48) becomes:

$$
\frac{x^{2}}{J^{\prime} / J_{1}^{\prime}}+\frac{y^{2}}{J^{\prime} / J_{2}^{\prime}}+\frac{z^{2}}{J^{\prime} / J_{3}^{\prime}}=1
$$

Equation (51) defines an ellipsoid whose semi-axis along the direction $x_{j}$ is $\sqrt{J^{\prime} / J_{j}^{\prime}} \cdot{ }^{3}$ Therefore, if we are at $P_{i}$, the semi-axis in the direction $x_{i}$ is

$$
\sqrt{\frac{J^{\prime}}{J_{i}^{\prime}}}>\sqrt{r_{i}}
$$

since $\Delta v^{2}>0$.

5.2.1. If $J_{1}^{\prime}>0$ then $\delta>2 / 3 I_{\alpha}$

$J_{1}^{\prime}>0$ implies $J_{2}^{\prime}>0$, and, as $J_{3}^{\prime}$ is always positive, Equation (51) defines an ellipsoid. From Equation (49), we know that the motion is only allowed inside this ellipsoid.

Suppose we are at $P_{i}$ so that $J^{\prime} / J_{i}^{\prime}>r_{i}$. Depending on the value of $J^{\prime} / J_{j \neq i}^{\prime}$ and $J^{\prime} / J_{k \neq i}^{\prime}$, the intersection between the two ellipsoids may change.

- If $J^{\prime} / J_{j}^{\prime} \geqslant r_{j}$ and $J^{\prime} / J_{k}^{\prime} \geqslant r_{k}$, then there is no intersection between the two ellipsoids and motion is allowed everywhere over the ellipsoid. A particle perturbed from $P_{i}$ has no barrier from traveling over the entire surface.

- If $J^{\prime} / J_{j}^{\prime}<r_{j}$ and $J^{\prime} / J_{k}^{\prime}<r_{k}$, then the intersection is an ellipse which encircles $P_{i}$ (Figure 1). $P_{i}$ is then Hill stable because the particle cannot leave $P_{i}$ without crossing a zero-velocity curve.

- If $J^{\prime} / J_{j}^{\prime}<r_{j}$ and $J^{\prime} / J_{k}^{\prime}>r_{k}$, then the intersection is an ellipse which encircles $P_{j}$ (Figure 2). A particle perturbed from $P_{i}$ may then reach $P_{k}$.

- If $J^{\prime} / J_{j}^{\prime}>r_{j}$ and $J^{\prime} / J_{k}^{\prime}<r_{k}$, then the intersection is an ellipse which encircles $P_{k}$. A particle perturbed from $P_{i}$ may then reach $P_{j}$.

$$
{ }^{3}\left(x_{1}, x_{2}, x_{3}\right)=(x, y, z)
$$




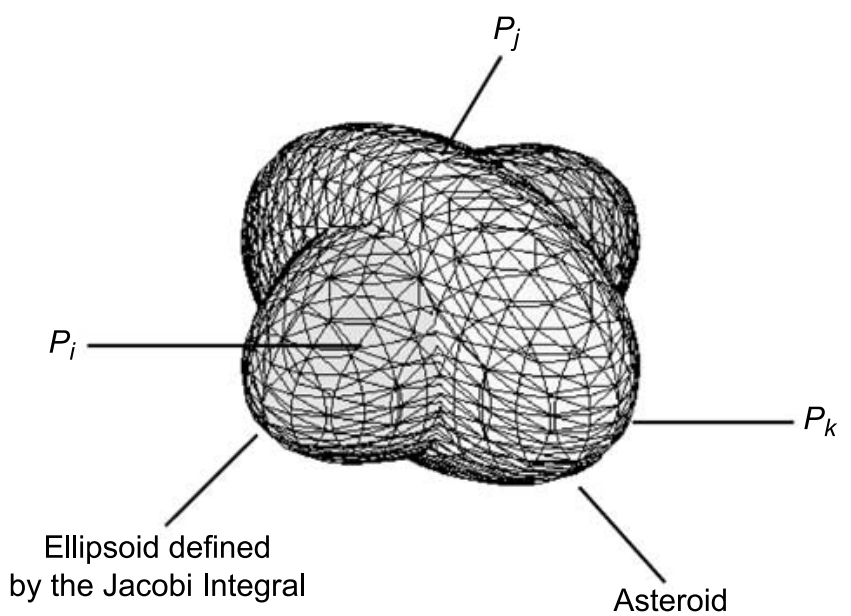

Figure 1. Intersection of the quadratic form defined by the Jacobi integral and the ellipsoid when $J^{\prime}=r_{i} J_{i}^{\prime}+\Delta J^{\prime}, J^{\prime} / J_{j}^{\prime}<r_{j}, J^{\prime} / J_{k}^{\prime}<r_{k}$ and $\delta>2 / 3 I_{\alpha}$.

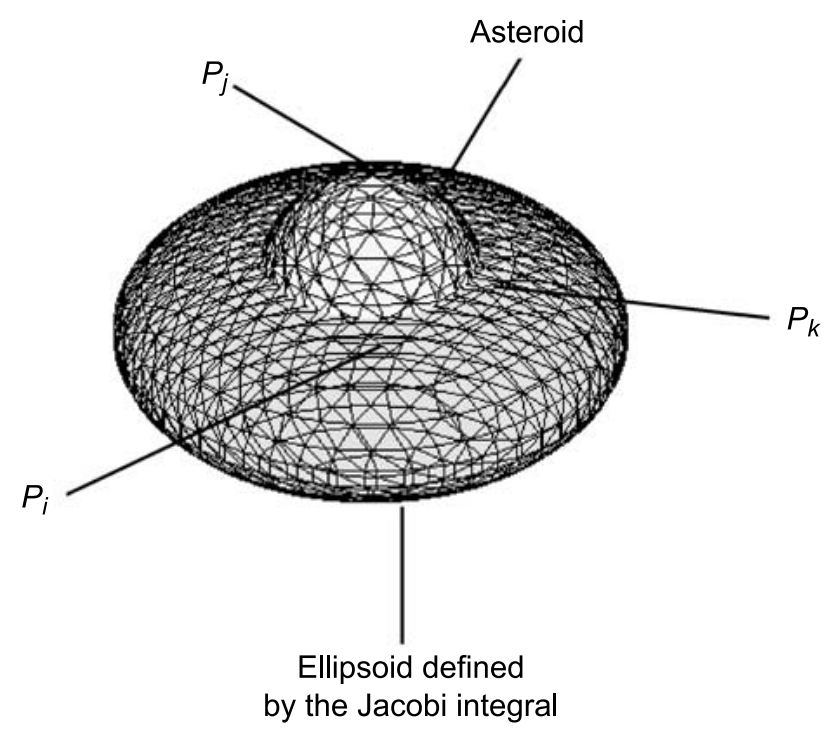

Figure 2. Intersection of the quadratic form defined by the Jacobi integral and the ellipsoid when $J^{\prime}=r_{i} J_{i}^{\prime}+\Delta J^{\prime}, J^{\prime} / J_{j}^{\prime}<r_{j}, J^{\prime} / J_{k}^{\prime}>r_{k}$ and $\delta>2 / 3 I_{\alpha}$.

So we conclude that:

- $P_{1}$ is Hill stable under the conditions:

$$
\begin{aligned}
& \frac{J^{\prime}}{J_{2}^{\prime}}<\beta^{2} \forall \Delta J^{\prime}>0 \Leftrightarrow \frac{J_{1}^{\prime}}{J_{2}^{\prime}}<\beta^{2}, \\
& \frac{J^{\prime}}{J_{3}^{\prime}}<\gamma^{2} \forall \Delta J^{\prime}>0 \Leftrightarrow \frac{J_{1}^{\prime}}{J_{3}^{\prime}}<\gamma^{2}
\end{aligned}
$$

These conditions are exactly Conditions 1 and 2 previously found. 
$-P_{2}$ is Hill stable under the conditions:

$$
\begin{gathered}
\frac{J^{\prime}}{J_{1}^{\prime}}<1 \forall \Delta J^{\prime}>0 \Leftrightarrow \frac{\beta^{2} J_{2}^{\prime}}{J_{1}^{\prime}}<1, \\
\frac{J^{\prime}}{J_{3}^{\prime}}<\gamma^{2} \forall \Delta J^{\prime}>0 \Leftrightarrow \frac{\beta^{2} J_{2}^{\prime}}{J_{3}^{\prime}}<\gamma^{2}
\end{gathered}
$$

These conditions are exactly Conditions 3 and 4.

$-P_{3}$ is Hill stable under the conditions:

$$
\begin{gathered}
\frac{J^{\prime}}{J_{1}^{\prime}}<1 \forall \Delta J^{\prime}>0 \Leftrightarrow \frac{\gamma^{2} J_{3}^{\prime}}{J_{1}^{\prime}}<1, \\
\frac{J^{\prime}}{J_{2}^{\prime}}<\beta^{2} \forall \Delta J^{\prime}>0 \Leftrightarrow \frac{\gamma^{2} J_{3}^{\prime}}{J_{2}^{\prime}}<\beta^{2}
\end{gathered}
$$

These conditions are exactly Conditions 5 and 6.

If any of the conditions are violated, we see that the particle not only loses Hill stability, but will have a natural motion away from $P_{i}$ along the unstable manifold.

5.2.2. If $J_{2}^{\prime}<0$ then $\delta<2 / 3 I_{\beta}$

$J_{2}^{\prime}<0$ implies $J_{1}^{\prime}<0$, thus, as $J_{3}^{\prime}$ is always positive, Equation (51) defines a hyperboloid.

1. If we are at $P_{3}$, then $J^{\prime}>0$ and the Jacobi integral defines a hyperboloid of two sheets:

$$
-\frac{x^{2}}{J^{\prime} /\left|J_{1}^{\prime}\right|}-\frac{y^{2}}{J^{\prime} /\left|J_{2}^{\prime}\right|}+\frac{z^{2}}{J^{\prime} / J_{3}^{\prime}}=1
$$

Motion is allowed everywhere over the ellipsoid (Figure 3), and $P_{3}$ is unstable.

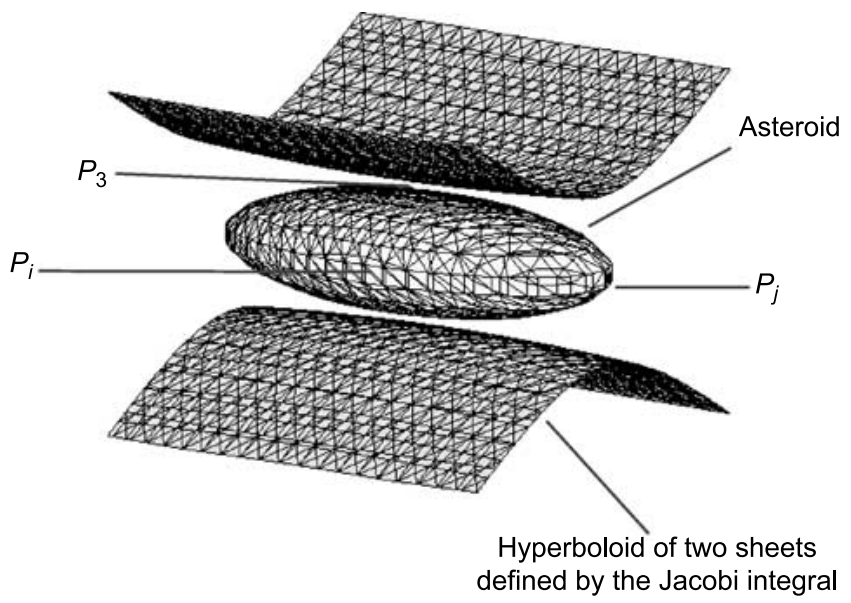

Figure 3. Intersection of the quadratic form defined by the Jacobi integral and the ellipsoid when $J^{\prime}=J_{3}^{\prime}+\Delta J^{\prime}$ and $\delta<2 / 3 I_{\beta}$. 


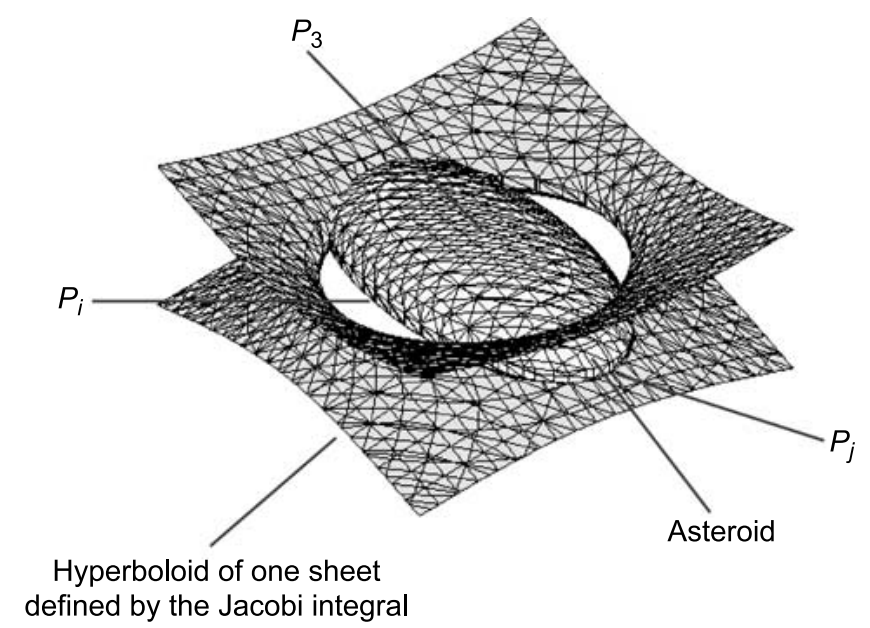

Figure 4. Intersection of the quadratic form defined by the Jacobi integral and the ellipsoid when $J^{\prime}=J_{i \neq 3}^{\prime}+\Delta J^{\prime}$ and $\delta<2 / 3 I_{\beta}$.

2. If we are at $P_{i \neq 3}$, then $J^{\prime}<0$ and the Jacobi integral defines a hyperboloid of one sheet:

$$
\frac{x^{2}}{\left|J^{\prime}\right| /\left|J_{1}^{\prime}\right|}+\frac{y^{2}}{\left|J^{\prime}\right| /\left|J_{2}^{\prime}\right|}-\frac{z^{2}}{\left|J^{\prime}\right| / J_{3}^{\prime}}=1
$$

At this point, we must pay attention to the meaning of $J^{\prime}<0$. In fact, by dividing Equation (49) by $J^{\prime}$, we change the inequality. The motion becomes allowed for $(x, y, z)$ such that:

$$
\frac{x^{2}}{\left|J^{\prime}\right| /\left|J_{1}^{\prime}\right|}+\frac{y^{2}}{\left|J^{\prime}\right| /\left|J_{2}^{\prime}\right|}-\frac{z^{2}}{\left|J^{\prime}\right| / J_{3}^{\prime}}>1
$$

which is outside the hyperboloid.

Thus the particle cannot be at $P_{i}$ (Figure 4), and $P_{i}$ does not exist.

So we conclude that $P_{3}$ is unstable in this case and that $P_{1}$ and $P_{2}$ do not exist.

\subsubsection{If $J_{2}^{\prime}>0$ and $J_{1}^{\prime}<0$ then $2 / 3 I_{\beta}<\delta<2 / 3 I_{\alpha}$}

1. If we are at $P_{1}$, then $J^{\prime}<0$ and the Jacobi integral defines a hyperboloid of two sheets (Figure 5):

$$
\frac{x^{2}}{\left|J^{\prime}\right| /\left|J_{1}^{\prime}\right|}-\frac{y^{2}}{\left|J^{\prime}\right| / J_{2}^{\prime}}-\frac{z^{2}}{\left|J^{\prime}\right| / J_{3}^{\prime}}=1
$$

We are in exactly the same case as Figure 3 (we just need to invert $P_{3}$ and $P_{1}$ ). Nevertheless, in that case $J^{\prime}<0$, therefore the motion is not allowed between the two sheets of the hyperboloid. The particle cannot be at $P_{1}$, and thus $P_{1}$ does not exist. 


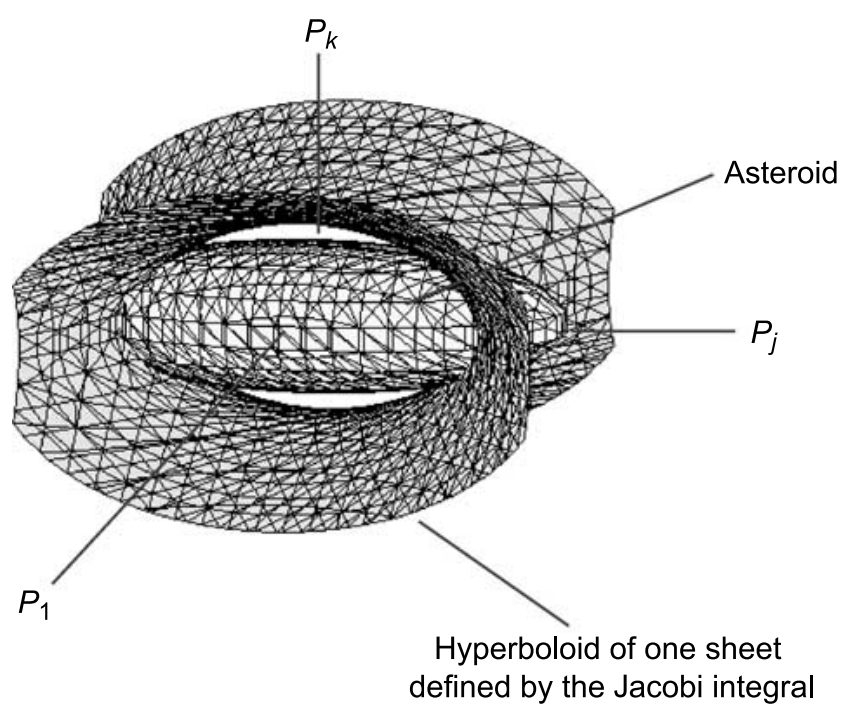

Figure 5. Intersection of the quadratic form defined by the Jacobi integral and the ellipsoid when $J^{\prime}=J_{k \neq 1}^{\prime}+\Delta J^{\prime}$ and $2 / 3 I_{\beta}<\delta<2 / 3 I_{\alpha}$.

2. If we are at $P_{k \neq 1}$, then $J^{\prime}>0$ and the Jacobi integral defines a hyperboloid of one sheet:

$$
-\frac{x^{2}}{J^{\prime} /\left|J_{1}^{\prime}\right|}+\frac{y^{2}}{J^{\prime} / J_{2}^{\prime}}+\frac{z^{2}}{J^{\prime} / J_{3}^{\prime}}=1
$$

The motion is allowed inside the hyperboloid, so that $P_{k}$ always exists but is unstable (Figure 5, the particle can always reach $P_{i}$ ).

So we conclude that $P_{2}$ and $P_{3}$ are unstable and $P_{1}$ does not exist.

Thus, we find again conditions similar to those of the previous section. Nevertheless, in that analysis we made an assumption on the smallness of deviations from $P_{i}$, whereas in the geometrical approach, we do not. We thus get a stronger stability, namely Hill stability. A crucial point to make is that loss of Hill stability is tied to loss of stability of the equilibrium points. Thus, once Hill stability is lost, linear instability of the equilibrium points is gained, allowing the particle to travel long distances across the ellipsoid. In the following, we will talk about stability as Hill stability.

\section{Conditions for the Existence and Stability of the Equilibrium Points}

\subsection{THE CONDITIONS FOR THE EXISTENCE OF EQUILIBRIUM POINTS}

First, let us summarize our results.

- If $\beta \neq 1$, we have found: 
1. Three types of equilibrium points, which satisfy these properties:

- $\delta<2 / 3 I_{\beta}, P_{1}$ and $P_{2}$ do not exist, $P_{3}$ is unstable.

- $2 / 3 I_{\beta}<\delta<2 / 3 I_{\alpha}, P_{1}$ does not exist, $P_{2}$ and $P_{3}$ are unstable.

- $2 / 3 I_{\alpha}<\delta, P_{1}$ is stable under Conditions 1 and $2, P_{2}$ is stable under Conditions 3 and 4 and $P_{3}$ is stable under Conditions 5 and 6.

2. For all $\beta$ a unique $(\gamma, \delta)$ defining the Jacobi ellipsoid exists, such that all the points on the surface are equilibrium points. These points are linearly unstable (Appendix A).

3. Three ellipses along the planes of symmetry may be in equilibrium under geometric constraints defined by Equations (20), (21) or (22). These equilibrium are linearly unstable along one direction.

- If $\beta=1$, we have found an infinite number of equilibrium points along the equator. The point $P_{3}$ always exists and is stable only under Conditions 5 and 6. All the other equilibrium points exist only if $\delta>2 / 3 I_{\alpha}$, but are linearly unstable along one direction at least.

It is also important to notice that replacing inequality by equality in Conditions 1, 2 and 4 lead to Equations (20)-(22), which defined the equilibrium figures.

\subsection{ANALYSIS OF THE CONDITION OF STABILITY}

The six conditions are summarized as

Condition 1: $\frac{3}{2} \delta\left(I_{\alpha}-\beta^{2} I_{\beta}\right)<1-\beta^{2}$,

Condition 2: $\frac{3}{2} \delta\left(I_{\alpha}-\gamma^{2} I_{\gamma}\right)<1$,

Condition 3: $\frac{3}{2} \delta\left(I_{\alpha}-\beta^{2} I_{\beta}\right)>1-\beta^{2}$,

Condition 4: $\frac{3}{2} \delta\left(\beta^{2} I_{\beta}-\gamma^{2} I_{\gamma}\right)<\beta^{2}$,

Condition 5: $\frac{3}{2} \delta\left(I_{\alpha}-\gamma^{2} I_{\gamma}\right)>1$,

Condition 6: $\frac{3}{2} \delta\left(\beta^{2} I_{\beta}-\gamma^{2} I_{\gamma}\right)>\beta^{2}$

First, we notice that two points cannot be stable at the same time. In fact, Condition 1 is opposite to Condition 3, Condition 2 is opposite to Condition 5, and Condition 4 and Condition 6 are also opposite. In all cases, we recall that $\delta>2 / 3 I_{\alpha}$.

The above conditions can be rewritten as
Condition $1: \Delta=\frac{2}{3 \delta}>C_{1}$,
Condition $2: \Delta=\frac{2}{3 \delta}>C_{2}$,
Condition $3: \Delta=\frac{2}{3 \delta}<C_{1}$,
Condition $4: \Delta=\frac{2}{3 \delta}>C_{3}$,
Condition $5: \Delta=\frac{2}{3 \delta}<C_{2}$,
Condition $6: \Delta=\frac{2}{3 \delta}<C_{3}$ 
where

$$
\begin{aligned}
& C_{1}=\frac{I_{\alpha}-\beta^{2} I_{\beta}}{1-\beta^{2}}=\int_{0}^{\infty} \frac{u \mathrm{~d} u}{(1+u)^{3 / 2}\left(\beta^{2}+u\right)^{3 / 2}\left(\gamma^{2}+u\right)^{1 / 2}}, \\
& C_{2}=I_{\alpha}-\gamma^{2} I_{\gamma}=\left(1-\gamma^{2}\right) \int_{0}^{\infty} \frac{u \mathrm{~d} u}{(1+u)^{3 / 2}\left(\beta^{2}+u\right)^{1 / 2}\left(\gamma^{2}+u\right)^{3 / 2}}, \\
& C_{3}=I_{\beta}-\frac{\gamma^{2}}{\beta^{2}} I_{\gamma}=\left(1-\frac{\gamma^{2}}{\beta^{2}}\right) \int_{0}^{\infty} \frac{u \mathrm{~d} u}{(1+u)^{1 / 2}\left(\beta^{2}+u\right)^{3 / 2}\left(\gamma^{2}+u\right)^{3 / 2}}
\end{aligned}
$$

We also know that a point cannot be stable if $\Delta>I_{\alpha}$.

\subsection{TOPOLOGY OF THE STABILITY CONDITIONS}

In order to analyze the different regions of stability we must plot the equations:

$$
\begin{aligned}
& \Delta=C_{1}(\beta, \gamma), \quad \Delta=C_{2}(\beta, \gamma), \quad \Delta=C_{3}(\beta, \gamma), \\
& \Delta=I_{\alpha}(\beta, \gamma)
\end{aligned}
$$

Figure 6 shows a representation of these equations.

The domain of the surface lies on a triangular region $0 \leqslant \gamma \leqslant \beta \leqslant 1$.

We can find exact results on the boundaries of this domain, $\beta=1, \gamma=\beta$ and $\gamma=0$.

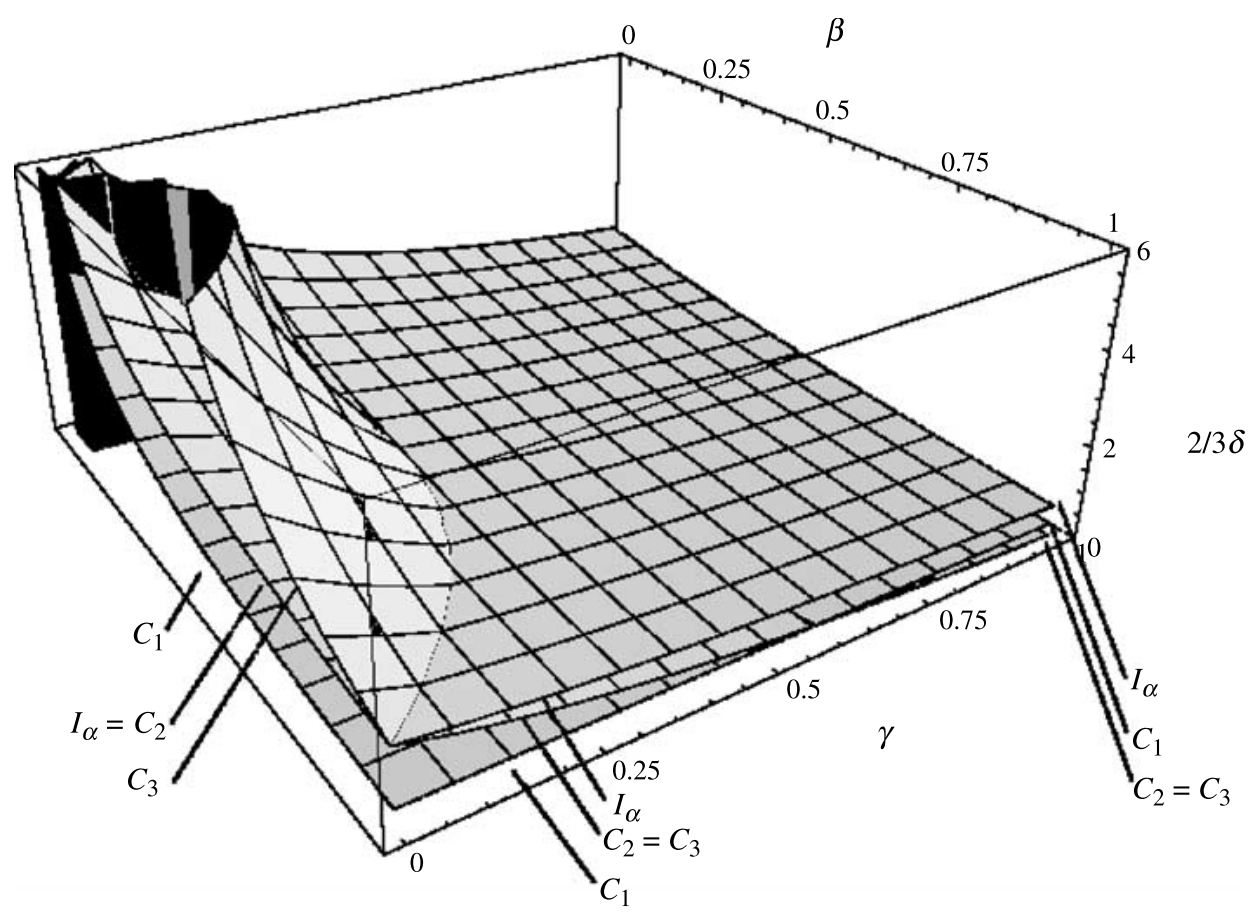

Figure 6. Representation of the conditions of stability. 
6.3.1. Conditions when $\beta=1$

We proved that all the points on the equator are unstable, and that $P_{3}$ is stable only under Conditions 5 and 6.

The stability boundaries are then:

$$
\begin{aligned}
& \Delta=C_{2}=\left(1-\gamma^{2}\right) \int_{0}^{\infty} \frac{u \mathrm{~d} u}{(1+u)^{2}\left(\gamma^{2}+u\right)^{3 / 2}}, \\
& \Delta=C_{3}=\left(1-\gamma^{2}\right) \int_{0}^{\infty} \frac{\mathrm{d} u}{(1+u)^{2}\left(\gamma^{2}+u\right)^{3 / 2}}, \\
& \Delta=I_{\alpha}=\int_{0}^{\infty} \frac{\mathrm{d} u}{(1+u)^{2}\left(\gamma^{2}+u\right)^{1 / 2}}
\end{aligned}
$$

Which can be integrated as

$$
\begin{aligned}
& \Delta=C_{2}=C_{3}=\frac{\left(2 \gamma^{2}+1\right) \arctan \left(\left(\sqrt{1-\gamma^{2}}\right) / \gamma\right)-3 \gamma \sqrt{1-\gamma^{2}}}{\left(1-\gamma^{2}\right)^{3 / 2}}, \\
& \Delta=I_{\alpha}=\frac{\arctan \left(\left(\sqrt{1-\gamma^{2}}\right) / \gamma\right)-\gamma \sqrt{1-\gamma^{2}}}{\left(1-\gamma^{2}\right)^{3 / 2}}
\end{aligned}
$$

These curves are plotted in Figure 7.

6.3.2. Conditions when $\beta=\gamma$

In this case $C_{3}$ is zero, so Condition 4 is always true and Condition 6 can never hold. The ellipsoid describes a prolate spheroid rotating about its short axis.

Thus $P_{3}$ is never stable and the conditions for stability of $P_{1}$ and $P_{2}$ are:

Condition $1 \Delta>C_{1}, \quad$ Condition $2 \Delta>C_{2}$,

Condition $3 \Delta<C_{1}$

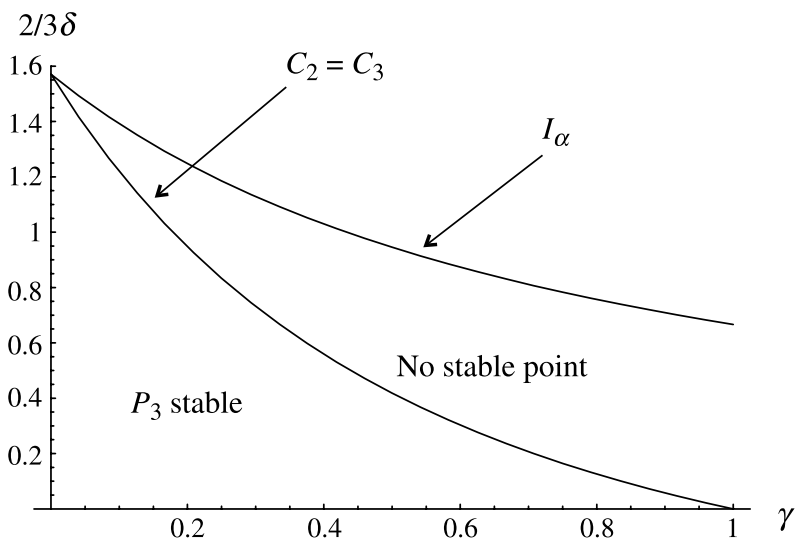

Figure 7. Topology of the stability conditions when $\beta=1$. 


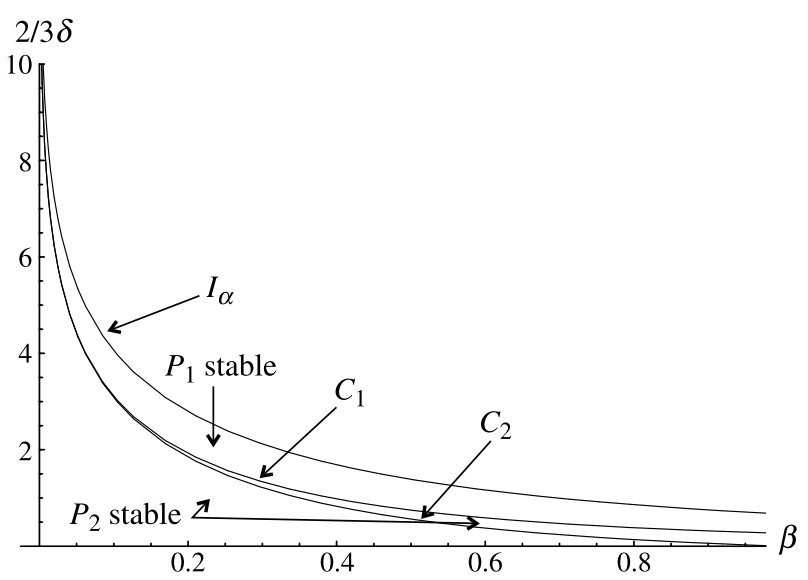

Figure 8. Topology of the stability conditions when $\beta=\gamma$.

The stability boundaries are:

$$
\begin{aligned}
& \Delta=C_{1}=\int_{0}^{\infty} \frac{u \mathrm{~d} u}{(1+u)^{3 / 2}\left(\beta^{2}+u\right)^{2}} \\
& \Delta=C_{2}=\left(1-\beta^{2}\right) \int_{0}^{\infty} \frac{u \mathrm{~d} u}{(1+u)^{3 / 2}\left(\beta^{2}+u\right)^{2}} \\
& \Delta=I_{\alpha}=\int_{0}^{\infty} \frac{\mathrm{d} u}{(1+u)^{3 / 2}\left(\beta^{2}+u\right)}
\end{aligned}
$$

Which can be integrated as

$$
\begin{aligned}
& \Delta=C_{1}=\frac{\ln \left[(1 / \beta)\left(1+\sqrt{1-\beta^{2}}\right)\right]\left(2+\beta^{2}\right)-3 \sqrt{1-\beta^{2}}}{\left(1-\beta^{2}\right)^{5 / 2}}, \\
& \Delta=C_{2}=\left(1-\beta^{2}\right) C_{1}, \\
& \Delta=I_{\alpha}=\frac{2 \ln \left[(1 / \beta)\left(1+\sqrt{1-\beta^{2}}\right)\right]-2 \sqrt{1-\beta^{2}}}{\left(1-\beta^{2}\right)^{3 / 2}}
\end{aligned}
$$

They are shown in Figure 8.

6.3.3. Conditions when $\gamma=0$

The ellipsoid shape describes a lamina with an elliptical shape in this case and the stability boundaries are then:

$$
\begin{aligned}
& \Delta=C_{1}=\int_{0}^{\infty} \frac{\sqrt{u} \mathrm{~d} u}{(1+u)^{3 / 2}\left(\beta^{2}+u\right)^{3 / 2}}, \\
& \Delta=C_{2}=\int_{0}^{\infty} \frac{\mathrm{d} u}{(1+u)^{3 / 2}\left(\beta^{2}+u\right)^{1 / 2} u^{1 / 2}},
\end{aligned}
$$




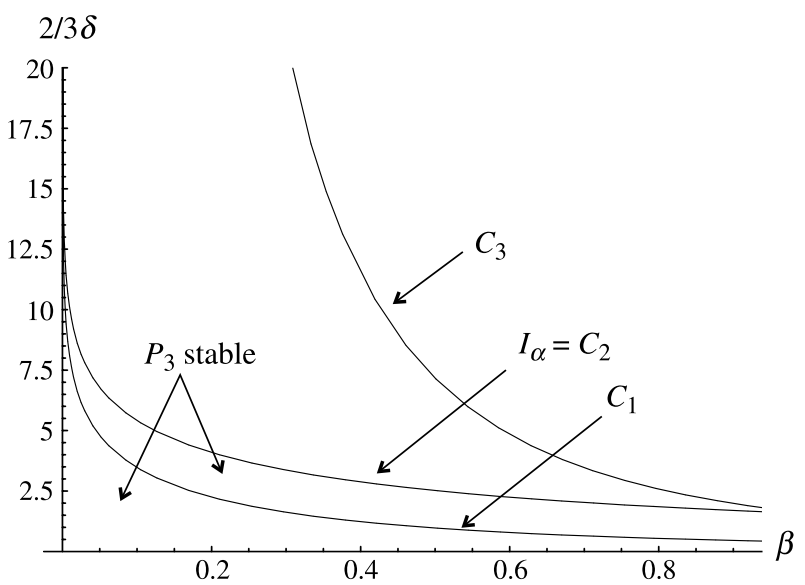

Figure 9. Topology of the stability conditions when $\gamma=0$.

$$
\begin{aligned}
& \Delta=C_{3}=\int_{0}^{\infty} \frac{\mathrm{d} u}{(1+u)^{1 / 2}\left(\beta^{2}+u\right)^{3 / 2} u^{1 / 2}}, \\
& \Delta=I_{\alpha}=\int_{0}^{\infty} \frac{\mathrm{d} u}{(1+u)^{3 / 2}\left(\beta^{2}+u\right)^{1 / 2} u^{1 / 2}}
\end{aligned}
$$

These are too difficult to integrate, but we notice that $C_{3}>C_{2}=I_{\alpha}>C_{1}$. Those inequalities allow us to conclude that only $P_{3}$ is stable. This result can also be seen by plotting the curves computed numerically (Figure 9).

6.3.4. Conditions when $\beta=1-\epsilon$ with $\epsilon \rightarrow 0$

As we noticed before, we expect a discontinuity in the properties of the equilibrium points when $\beta$ reaches 1 .

Therefore, when $\beta=1-\epsilon$, the drawing of the conditions must be very different.

We clearly see in Figure 10 that each condition can be met. When $\epsilon \rightarrow 0$ Conditions $C_{2}$ and $C_{3}$ become equivalent, and as studied before, when $\epsilon$ reaches 0 the equilibrium points are all unstable.

\subsection{INTERPRETATION}

Now we can make some preliminary conclusions.

As $C_{2}$ and $C_{3}$ are always positive:

$\forall(\beta, \gamma), \beta \neq 1 \exists \Delta$ small enough $\mid P_{3}$ is stable

As $C_{1}$ and $C_{2}$ are always smaller than $I_{\alpha}$ :

$\forall(\beta, \gamma), \beta \neq 1 \exists \Delta$ big enough $\mid P_{1}$ is stable

The larger $\gamma$ is, the larger is the space where $P_{1}$ is stable and the smaller is the one of $P_{3}$. 


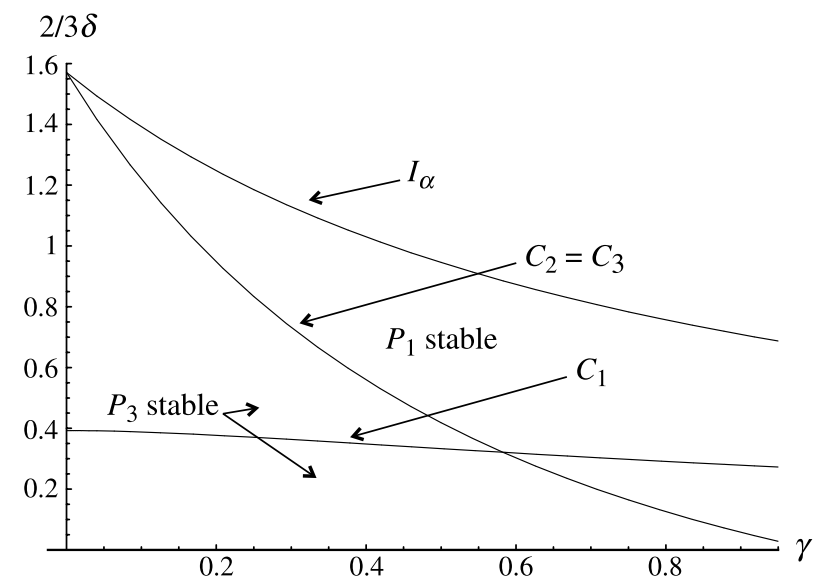

Figure 10. Topology of the stability conditions when $\beta=1-\epsilon$ with $\epsilon \rightarrow 0$.

Thus, when $\gamma=0$ only $P_{3}$ is stable, and when $\gamma=\beta, P_{3}$ is never stable. Nevertheless, when $\beta=1$ all the points on the equator are unstable equilibrium points.

Concerning $P_{2}$, the analysis is more complicated because its area of stability is intermediate between $P_{1}$ and $P_{3}$.

Here is the condition:

$$
\forall(\beta, \gamma)\left|C_{3}-C_{1}<0 \exists \Delta\right| P_{2} \text { is stable }
$$

Now let us discuss when $C_{3}-C_{1}<0$.

6.4.1. The necessary condition of stability for $P_{2}: C_{3}-C_{1}<0$

The inequality we must study is

$$
\int_{0}^{\infty} \frac{\left(1-\gamma^{2}\right)-\left(\gamma^{2} / \beta^{2}\right)(1+u)}{(1+u)^{3 / 2}\left(\beta^{2}+u\right)^{3 / 2}\left(\gamma^{2}+u\right)^{3 / 2}} \mathrm{~d} u<0
$$

An analytical analysis is not possible because of the complexity of the integral. Nevertheless, we can compute it numerically (Figure 11).

First of all we notice that $P_{2}$ cannot always be stable. When $\beta$ and $\gamma$ are close to each other then $P_{2}$ may be stable under the condition of existence and Conditions 3 and 4 . These conditions can be interpreted as a condition on the curvature of the ellipsoid, if it is not 'round enough', $P_{2}$ cannot be stable. We can further simplify the interpretation of these results. In fact, as there exists a unique solution to the equations $C_{1}=C_{2}=C_{3}$ the space $(\beta, \gamma)$ is divided into two regions (Figure 12). ${ }^{4}$

${ }^{4}$ These equations define the MacLaurin spheroids and the Jacobi ellipsoids. More details are given in Appendix A. 

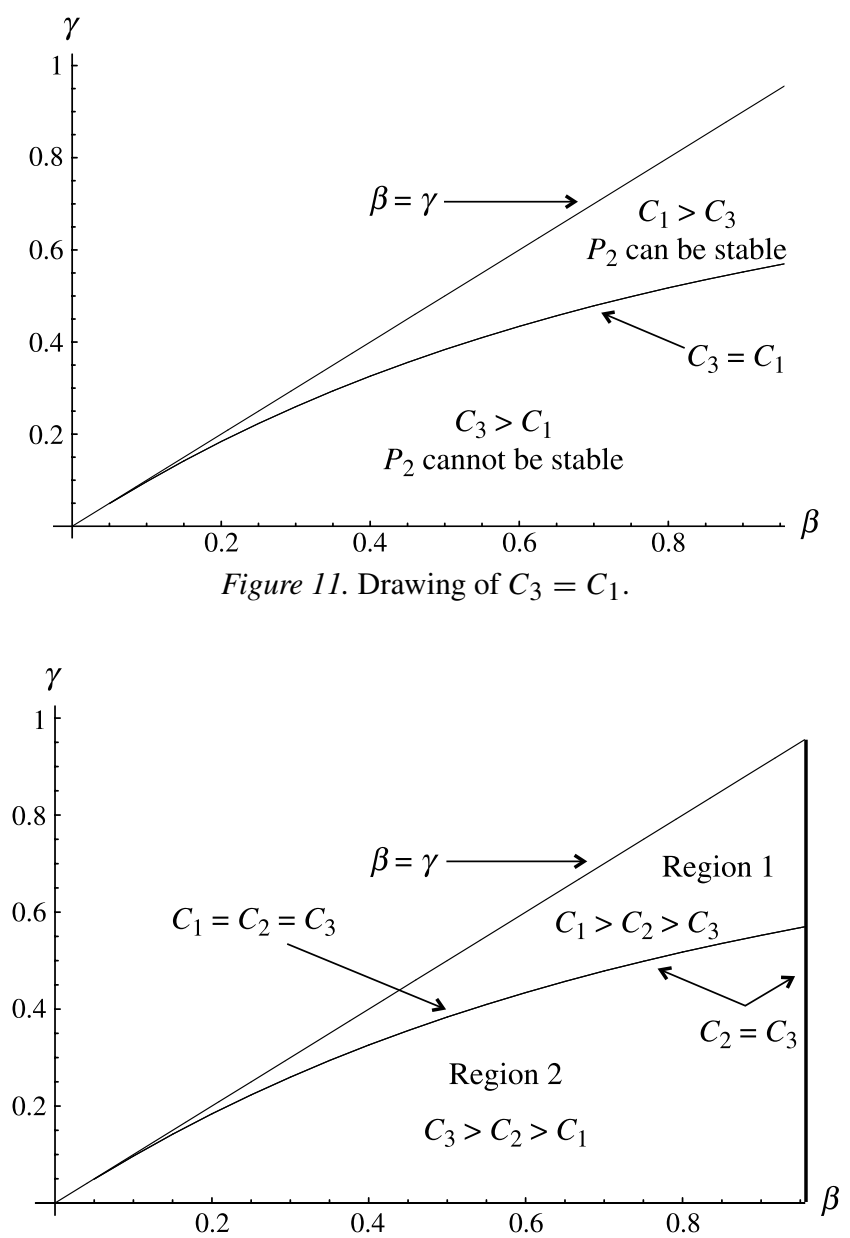

Figure 12. Drawing of $C_{1}=C_{2}, C_{1}=C_{3}$ and $C_{2}=C_{3}$.

In Region 1, the conditions of stability become:

$$
\begin{aligned}
& \Delta>C_{1} \Rightarrow P_{1} \quad \text { stable }, \\
& C_{1}>\Delta>C_{3} \Rightarrow P_{2} \quad \text { stable }, \\
& C_{3}>\Delta \Rightarrow P_{3} \quad \text { stable }
\end{aligned}
$$

$C_{2}$ does not play any role in this region. In Region 2, the conditions of stability become:

$$
\Delta>C_{2} \Rightarrow P_{1} \quad \text { stable, } \quad C_{2}>\Delta \Rightarrow P_{3} \quad \text { stable }
$$

Only $C_{2}$ is relevant in this case. 
6.4.2. What happens when crossing one of the surfaces $\Delta=C_{1}$,

$\Delta=C_{2}$ or $\Delta=C_{3}$

When $\Delta=C_{1}, \Delta=C_{2}$ and $\Delta=C_{3}$ are concurrent, their intersection is a line in the three-dimensional space $(\beta, \gamma, \Delta)$. As we noticed in the beginning of this section, this line defines the Jacobi ellipsoid (or the MacLaurin spheroid if $\beta=1$ ).

Each surface $\Delta=C_{i}$ corresponds to the geometry for which all points on the ellipse defined by this equation are in equilibrium. It is now obvious that this case includes, but is not restricted to, the Jacobi ellipsoids or MacLaurin spheroids.

Using these remarks, we can explain how the stability evolves as $\Delta$ increases.

When $\Delta$ is close to zero, $P_{3}$ is always stable. Let $\Delta$ increase.

- If $\gamma$ is big enough to be in Region 1 , we will first cross $C_{3}$. When crossing this surface, all points on the ellipse which lies in the plane $x=0$ are in equilibrium. They are linearly unstable along a tangent direction to the ellipse but are stable along the $x$-axis. Then, above $C_{3}, P_{2}$ is stable. We then cross $C_{2}$ but as we saw before this surface has no meaning in this region. Finally we cross $C_{1}$. When crossing this surface, all points on the ellipse which lies in the plane $z=0$ are in equilibrium and are stable along the $z$-axis but linearly unstable along the other axis (if $\beta$ and $\gamma$ are small enough the ellipse may not exist since $C_{3}$ may be above $\Delta=I_{\alpha}$ ). As $\Delta$ increased, after $P_{1}$ is stable, we then cross $\Delta=I_{\alpha}$, and the equilibrium points do not exist anymore or are unstable.

- If $\gamma$ is small enough to be in Region 2, the only relevant surface is $C_{2}$. When we are crossing $C_{2}$, all points on the ellipse which lie in the $y=0$ plane are in equilibrium and they are linearly unstable along a tangent direction to the ellipse and stable along the $y$-axis. Above this surface, $P_{1}$ is stable.

- If we cross all the surfaces at the same time, that is, when $\Delta$ is such that the ellipsoid is a Jacobi ellipsoid or a MacLaurin spheroid, then all the points on the surface are in equilibria and are linearly unstable. Above this surface, $P_{1}$ is stable.

\section{Some Examples}

Now we apply our results to some well-known asteroids: We assume the density to be $2.5 \mathrm{~g} / \mathrm{cm}^{3}$ except for Eugenia and Mathilde whose density are $1.7 \mathrm{~g} / \mathrm{cm}^{3}$ and Kleopatra whose density is $7.0 \mathrm{~g} / \mathrm{cm}^{3}$.

Our data are taken from [3]. We use the synthesis values of data when they exist, elsewhere we calculate an average value of all observations of each asteroid.

Most of the asteroids have an equilibrium point which is stable. Two asteroids, Siwa and Chiron, are spheres, so they do not have any stable points. Two asteroids, Icarus and Apollo, have only equilibrium points $P_{2}$ and $P_{3}$ (as we saw before, it must be $P_{1}$ which does not exist). 
Among the 66 asteroids which have one stable equilibrium point, 3 have $P_{1}$ stable, 39 have $P_{2}$ stable and 24 have $P_{3}$ stable.

These results show that, a priori, all the different geometry models we just studied are liable to exist. Nevertheless, due to the lack of accuracy in our data (especially the density), our results for specific asteroids may change, but they will remain true from a statistical point of view.

\section{Conclusions}

We study the stability of equilibrium points on the surface of a rotating ellipsoid. We find that there are six equilibrium points for a general ellipsoid (each symmetric about the origin) with only two symmetric points stable at any given time. If the ellipsoids are Jacobi or MacLaurin ellipsoids, then all points on the surface are at equilibrium, albeit unstable. We find that the stability properties of the surface equilibria change as a function of 'where' the ellipsoid lies in relation to the classical figures of equilibriums. We show that some 'measured' asteroid shapes exhibit the more exotic case of stability along the long axis of the rotating ellipsoid.

\section{Appendix A. MacLaurin Spheroids and Jacobi Ellipsoids}

The aim of this appendix is to link our work with the study of Chandrasekhar [1] on the ellipsoidal figures of equilibrium.

Let us consider a fluid rotating about its $z$-axis, and let us write the conditions for the fluid to be in equilibrium. Using Chandrasekhar's notation we find in Equation (17), [1, p. 6]:

$$
2 A_{1} a_{1}^{2}-\frac{\Omega^{2}}{\pi G \rho} a_{1}^{2}=2 A_{2} a_{2}^{2}-\frac{\Omega^{2}}{\pi G \rho} a_{2}^{2}=2 A_{3} a_{3}^{2}
$$

with

$$
a_{1}=\alpha, \quad a_{2}=\beta, \quad a_{3}=\gamma, \quad A_{i}=a_{1} a_{2} a_{3} I_{\alpha}
$$

The first equality is exactly Equation (20) and the second equality is the same as Equation (21).

In his book, Chandrasekhar shows that these equations admit only one solution. The solution is known as the MacLaurin spheroids in the case $\beta=1$ and the Jacobi ellipsoids otherwise.

From the definition of these figures, we deduce that each point is then in equilibrium but none of them are stable (indeed they are all linearly unstable). Obviously these conclusions may be applied to our study. 


\section{References}

1. Chandrasekhar, S.: 1969, Ellipsoidal Figures of Equilibrium, Yale University Press, New Haven, CT.

2. Chauvineau, B., Farinella, P. and Mignard, F.: 1993, 'Planar orbits about a triaxial body: applications to asteroidal satellites', Icarus 105, 370-384.

3. http://pdssbn.astro.umd.edu/SBNast/holdings/EAR-A-5-DDR-ASTEROID-SPIN-VECTORSV4.0.html.

4. Danby, J. M. A.: 1988, Fundamentals of Celestial Mechanics, 2nd edn, Willmann-Bell.

5. Hagihara, Y.: 1970, Celestial Mechanics, Dynamical Principles and Transformation Theory, Vol. I, MIT Press, Cambridge, MA.

6. Hudson, R. S., Ostro, S. J., Jurgens, R. F., Rosema, K. D., Giorgini, J. D., Winkler, R., Rose, R., Choate, D., Cormier, R. A., Franck, C. R., Frye, R., Howard, D., Kelley, D., Littlefair, R., Slade, M. A., Benner, L. A. M., Thomas, M. L., Mitchell, D. L., Chodas, P. W., Yeomans, D. K., Scheeres, D. J., Palmer, P., Zaitsev, A., Koyama, Y., Nakamura, A., Harris, A. W. and Meshkov, M. N.: 2000, 'Radar observations and physical modeling of asteroid 6489 Golevka', Icarus 148, 37-51.

7. MacMillan, 1930, The Theory of the Potential, McGraw-Hill, New York.

8. Ostro, S., Hudson, R., Benner, L., Nolan, M., Giorgini, J., Scheeres, D. J., Jurgens, R. and Rose, R.: 2001, 'Radar observations of asteroid 1998 ML14, Meteoritics Planetary Sci. 36(9), $1225-1236$.

9. Robinson, M. S., Thomas, P. C., Veverka, J., Murchie, S. and Carcich, B.: 2001, 'The nature of ponded deposits on Eros', Nature 413, 396-400.

10. Sawai, S., Kawaguchi, J., Scheeres, D. J., Yoshizawa, N. and Ogasawara, M.: 2001, 'Development of a target marker for landing on asteroids', J. Spacecraft Rockets 38(4), 601-608.

11. Scheeres, D. J.: 1994, 'Dynamics about uniformly rotating triaxial ellipsoids: application to asteroids', Icarus 110, 225-238.

12. Scheeres, D. J., Ostro, S. J., Hudson, R. S. and Werner, R. A.: 1996, 'Orbits close to asteroid 4769 Castalia', Icarus 121, 67-87.

13. Scheeres, D. J., Ostro, S. J., Hudson, R. S., DeJong, E. M. and Suzuki, S.: 1998, 'Orbit dynamics about 4179 Toutatis', Icarus 132, 53-79.

14. Szebehely, V.: 1984, 'Review of concepts of stability', Celest. Mech. \& Dyn. Astr. 34, 49-60.

15. Werner, R. A. and Scheeres, D. J.: 1997, 'Exterior gravitation of a polyhedron derived and compared with harmonic and mascon gravitation representations of asteroid 4769 Castalia', Celest. Mech. \& Dyn. Astr. 65, 313-344.

16. Zuber, M. T., Smith, D. E., Cheng, A. F., Garvin, J. B., Aharonson, O., Cole, T. D., Dunn, P. J., Guo, Y., Lemoine, F. G., Neumann, G. A., Rowlands, D. D. and Torrence, M. H.: 2000, 'The shape of 433 Eros from the NEAR-Shoemaker Laser Rangefinder', Science 289, 2097-2101. 